\title{
Nuclear and Particle Physics
}

\section{University of Virginia}

\section{Quasielastic and Deep Inelastic Electron Scattering at High Energies}

\author{
DONAL B. DAY \\ Institute of Nuclear and Particle Physics and Department of Physics \\ University of Virginia \\ Charlottesville, VA 22901 USA
}

\section{DISCLAIMER}

Thjs report was prepared as an account of work sponsored by an agency of the United States Government. Neither the United States Governmert nor any agency thereof, nor any of their employees, makes any warranty, express or implied, or assumes any legal liability or responsibility for the accuracy, completeness, or usefulness of any information, apparatus, product, or process disclosed, or represents that its use would not infringe privately owned rights. Reference herein to any specific commercial product, process, or service by trade name, trademark, manufacturer, or otherwise does not necessarily constitute or imply its endorsement, recommendation, or favoring by the United States Government or any agency thereof. The views and opinions of authors expressed herein do not necessarily state or reflect those of the United States Government or any agency thereof.

$$
\text { FGO5-86ER40261 }
$$

Invited talk presented at the

\section{Second European Workshop on}

Hadronic Physics With Electrons Beyond $10 \mathrm{GeV}$

Dourdan, France

Dctober 8-13. 1990 


\title{
Inclusive Quasielastic and Deep Inelastic Electron Scattering at High Energies
}

\author{
Donal B. Day
}

Institute of Nuclear and Particle Physics

University of Virginia

Charlottesville, VA 22901

\begin{abstract}
With high electron energies a kinematic regime can be reached where it will be possible to separate quasielastic and deep inelastic scattering. We present a short description of these pracesses which dominate the inclusive spectrum. Using the highest momentum transfer data available to guide our estimates, we give the kinematic requirements and the cross sections expected. These results indicate that inclusive scattering at high $q$ has a yet unfilled potential.
\end{abstract}

\section{Introduction}

At high momentum transfers it is possible to describe the inclusive electron scattering spectrum in terms of two distinct processes. The first (See Figure 1a) is the absorption of a virtual photon by an off-shell nucleon in the nucleus (quasielastic scattering); with
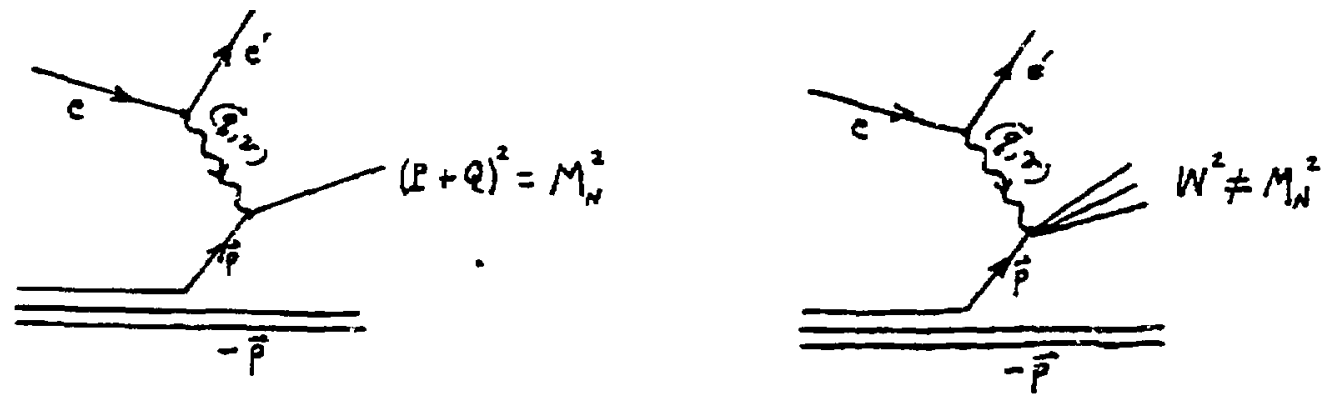

Figure 1: The Feynman diagrams of the dominant processes at large momentum transfer: a) Quasielastic knockout of single nucleons and b) deep inelastic scattering from nucleons. 


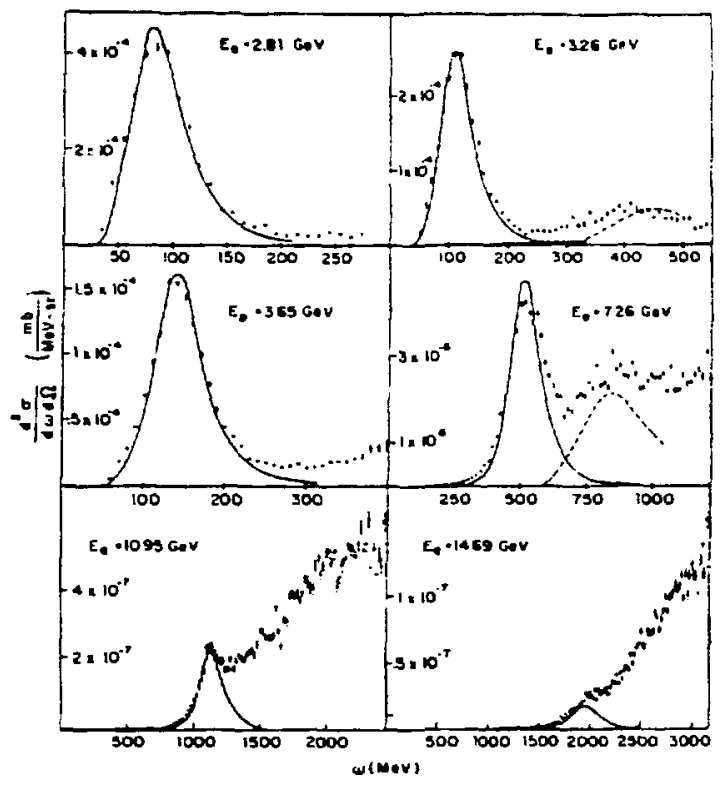

Figure 2: Inclusive data [2] on ${ }^{3} \mathrm{He}$ at $8^{\circ}$ and six different energies.

the transfer of momentum $\vec{q}$ and energy $\boldsymbol{\omega}$ the nucleon is ejected from the nucleus with the constraint that $P_{m}^{2}=M_{n}^{2}$, i.e. the final knocked' out nucleon is on it's mass shell. This mechanism is responsible for the broad quasielastic peak seen in the spectrum of final electron energies and is a source of information about the momentum and energy distributions of the bound nucleons [i]. Quasielastic scattering is characterized by the momentum dependence of the elastic nucleon form factor - the cross section falls like $1 / Q^{8}$. The second process (Figure $1 b$ ) is inelastic scattering from bound off-shell nucleons in the nucleus with an indeterminate final state. This process includes resonance production and non-resonant pion production from nucleons; it is dominated by the deep inelastic scattering from the nucleon constituents (quarks). At large $Q^{2}$ and $\omega$ data exhibit Bjorken scaling with only a QCD logarithmic dependence on $\mathrm{Q}^{2}$ and can be expressed in terms of the distribution function of the quarks inside the nucleus directly.

The two processes share the same initial state nuclear structure as can be seen from the Feynman diagrams. In the impulse approximation, they can both be expressed as a convolution of the nuclear spectral function with the appropriate elastic and inelastic electron-nucleon structure, constrained only by energy and momentum balance. Unfortunately the transition from one process to the other is a smooth one and it is impossible to use the dependence on energy transfer to separate them experimentally. However it is possible to exploit the momentum dependence as a vernier to control the relative dominance of the two processes. In Figure 2 we show inclusive data $[2]$ from ${ }^{3} \mathrm{He}$ as an example of the gradual transition from the dominance of quasielastic scattering to deep inelastic scattering. At 'low' momentum transfers the spectrum is dominated by a large quasifree peak centered at approximately at $\omega=Q^{2} / 2 m_{n}$. As the incident energy increases (these data were taken at a fixed electron scattering angle so that the momentum transfer increases with incident electron energy) we see the gradual growth of deep inelastic 
contribution that eventually obscures the quasielastic peak. By measuring the inclusive spectra at differcnt momentum transfers we observe the transition from the quasieiastic case (1a) to the inelastic case (1b).

There is obvious interest in studying these processes. The interaction of the virtual photon with a moving nucleon inside the nucleus can provide an indirect measure of the nucleon momentum and energy distribution. Of particular interest are the high- $k$ components which can only be described by going beyond a single particle mean field description of the nucleus. The high momentum strength is directly related to the existence of NN correlations. Second, the interaction exposes the fundamental electron-nucleon vertex. In quasielastic scattering this is the elastic (off-shell) electron-nucleon interaction. When the spectrum is dominated by deep inelastic scattering the vertex is described by scatterings from the individual quarks and the $x$ behavior (in the scaling region) is an direct measure of the parton momentum distribution. The extensive experimental and theoretical studies of the role of the nuclear medium on the inelastic structure functions (the EMC effect) have been limited to the $x<1$. It will be possible with high incident energies to extend the experimental studies into the region of large $x(\geq 1)$.

The rest of this paper is organized as follows. In section 2 we will give a brief introduction to $y$ scaling, which with certain assumptions, can provide a direct measure of the nucleon momentum distribution. Examples will be discussed. In section 3 the deep inelastic contribution to the inclusive spectra will be presented in terms of conventional nuclear physics. Calculations of DIS are compared to data so as to gain some confidence about our understanding of this region. Finally in the Section 4 we will give the kinematics necessary to explore the transition region between quasielastic and deep inelastic scaitering at $x \geq 1$. Count rates based on a conventional calculation will be also given.

\section{Quasielastic Scattering and Scaling}

At high $q$ and $\omega$ the interaction of the virtual photon with the nucleus can be described by the impulse approximation in which the nucleons are considered 'free' particles. By that we mean the final state includes only the free nucleon which has been knocked out, and the recoiling $(A-1)$ system. We do not consider excitation of the nucleon or interaction of the virtual photon with charge and current carrying pions in the nucleus. In addition we assume that the knockef out nucleon has no interaction with the residual nucleus. In inclusive measurements, one has no control over these processes and they do contribute to the measured cross sections in various amounts depending on the kinematics.

In the PWIA [3] the quasielastic cross section can be written

$$
\begin{aligned}
\frac{d^{2} \sigma}{d \omega d \Omega_{e^{\prime}}}= & \sum_{i=1}^{A} \int d \vec{k} \int d E \sigma_{e i} S_{i}(E, k) \\
& \times \delta\left(\omega-E+M_{A}-\left(M^{2}+\vec{k}^{\prime 2}\right)^{1 / 2}-\left(M_{A-1}^{2}+\vec{k}^{2}\right)^{1 / 2}\right),
\end{aligned}
$$

where $\vec{k}$ and $\overrightarrow{k^{\prime}}=\vec{k}+\vec{q}$ are the initial and final momenta of the struck nucleon. $S_{i}(E, k)$, the spectral function, is the combined probability to find a nucleon ( $i=$ proton or neutron) of momentum $k$ and energy $E$ in the nucleus. $M_{A}$ is the mass of the nucleus, $M_{A-1}$ the 
mass of the residual nucleus, and $E$ the separation energy. (Removal of a nucleon from the nucieus requires an energy $E$ which may leave the residual nucleus in an excited state.) $\sigma_{e i}$ is the relativistic (off-shell) electron-nucleon cross section for a moving nucleon [4] and is a function of $q, \omega, \vec{k}$ and $E$.

From the energy momentum balance it should not be too difficult to convince oneself that inclusive measurements receive contributions from a range of momenta and separation energies of the spectral function, $S\left(E_{s}, k\right)$. However, the cross section in the maximum is dominated by $S(E, k=0)$, the small energy loss side of the peak by high $k$, small $E$ components in the spectral function.

\section{$2.1 Y$ Scaling}

In $1975 \mathrm{G}$. West [5] suggested that ai high momentum transfer, quasielastic electron scattering would become the function of a single variable, $y$, the component of the initial nucleon momentum parallel to $\vec{q}$. At high momentum transfer, West suggested, one could measure directly the momentum distribation of nucleons inside the nucleus. Since 1975, a significant effort has gone into refining the understanding of $y$ scaling. Here we will simply use the results of these studies and refer the reader to some recent reviews $[6,7]$.

In the PWIA the quasielastic electron-nucleus cross section can be reduced to product of a scaling function, $F(y)$, which is directly related to the momentum distribution $n(k)$, and the elementary electron-nucleon cross section which is known from elastic scattering. Briefly, the PWIA (with some additional assumptions about isospin independence of the spectral function and the dependence of the elementary cross section on $(k, E)$ ), allows Eq. 1 to be written as

$$
\frac{d^{2} \sigma}{d \Omega_{e} d \omega} \cong\left(Z \tilde{\sigma}_{e p}+N \tilde{\sigma}_{e n}\right) \cdot K \cdot F(q, y)
$$

where

$$
F(q, y)=2 \pi \int_{-y}^{Y} k d k \int_{E_{\min }}^{E_{\max }(q, y)} d E S(k, E)
$$

$K$ is a kinematic factor coming from the integration over the angle between $\vec{q}$ and $\ddot{k}$. The definition of $y$ comes from the solution of the delta function in Eq. 1 for the minimum value of $E$ and for a given $q$ and $\omega$ can be found from

$$
\omega=\omega(q, y)=\sqrt{M^{2}+(q+y)^{2}}+\sqrt{M_{A-1}^{2}+y^{2}}-M_{A} .
$$

$y$ is negative for energy loss $\omega<\frac{Q^{2}}{2 M_{n}}$. For $q$ sufficiently large, $Y \rightarrow \infty$ and $E_{\text {max }}$ becomes independent of $q$ and goes to $E_{M}^{\rho} \neq \infty$. Thus $F(q, y) \rightarrow F(\infty, y) \equiv F(y)$ and the right hand side of Eq. 2 divided by the elementary electron-nucleon cross section and a kinematic factor becomes a function independent of $q$. Recalling that the momentum dis $-i$ ibution is just

$$
n(k)=\int_{E_{\min }}^{\infty} d E S(k, E)
$$

we immediately see the relation of the scaling function $F(q, y)$ to the momentum distribution:

$$
F(q, y) \equiv 2 \pi \int_{-y}^{Y} k d k \bar{n}(q, y ; k)
$$




$$
\bar{n}(g, y ; k) \equiv \int_{E_{\min }}^{E_{M}} d E S(k, E)<\bar{\pi}(\infty, y, k)<n(\dot{k}) .
$$

Since the kinematics of electron scattering forbid us from doing the complete integral (Eq. 3) we can not conclude that $\bar{n}(q, y, k)$ is the nucleon momentum distribution without some model for the energy distribution of the spectral function. Only in the special case of the deuteron where $S(k, E)$ has all the strength located at a single value of $E$ or when the strength is lecalized at small values of $E$ can we be confident that the scaling analysis at large $q$ would return the nucleon momentum distribution. For nuclei with $A>2$ we expect the saturation of this integral at moderate values of $y$, while at very large initial momenta the residual system should be highly excited. This would imply that momentum distribution extracted from large $q$ data would be a lower limit on the nucleon momentum distribution. This point is emphasized through Figures $3 \mathrm{a}$ and $3 \mathrm{~b}$ where we show the $\mathrm{T}$ $=0$ piece of the ${ }^{3} \mathrm{He}$ spectral function [8] (the solid line at $E=0$ is the two-body breakup divided by 10 ) along with the integration limits of Eq. 3 shown in $(k, E)$ plane. The top part of Figure 3 is for $y=-0.2 \mathrm{GeV} / \mathrm{c}$ for momentum transfer $q=0.5,1.0,1.5$, 2.0 , and $100 \mathrm{GeV} / \mathrm{c}$. At $y=-0.2 \mathrm{GeV} / \mathrm{c}$ the integration region grows very quickly with momentum transfer and at finite values of $q$ we can expect that the dominant piece of the spectral function has been integrated. Figure 3 (bottom) shows that at larger values of initial nucleon momenta, $y=-0.5 \mathrm{GeV} / \mathrm{c}$, even at large values of $q$ it is not possible to integrate over the entire piece of the spectral function that contributes to $n(k)$. This constitutes a serious drawback for the model independent interpretation of the scaling function in terms of $n(k)$. Attempts have been made by the Rome group $[9,7]$ to account for these so called "binding corrections". Fortunately, as they have shown, it is possible to circumvent this problem at the risk of introducing some small model dependence.

Note that in PWIA, the scaling function, $F(q, y)$ at fixed $y$ and variable $q$, reaches it's large $q$ limit from below; as $q$ increases, the upper limits of the momentum and energy integral grow. Any other behavior indicates a violation of PWIA (which in fact we see in the experimental data).

\subsection{Examples}

Application of this scaling hypothesis to the avaliable data forces a strong impression of its general validity. For example, a set of high $q$ data on ${ }^{4} \mathrm{He}[10,11,12]$ is presented in terms of the scaling function $F(q, y)$ in Figure 4. The cross sections (not shown) range over 5 orders of magnitude and the position of the quasielastic peak varies from 0.2 to 1.5 $\mathrm{GeV}$ in energy loss. For values of $y$ less than zero the data sets describe a narrow band in the scaiing function. At positive $y$, the presence of inelastic contributions to the cross section destroys the scaling. This should be expected since those processes (e.q. real and virtual pion production) are not described by Eq. 1. The positive $y$ region is shown in detail in Figure 5 in order to emphasize this point. This region includes the $\Delta$ whose position is not fixed in $y$ as the momentum transfer changes. The insert in Figure 4 gives the convergence of the scaling function as a function of momentum transfer. Note that it approaches the asymptotic values from above, in contradiction to the expectation of the PWIA.

The source of this violation is strongly hinted to by the behavior of heavy nuclei which 

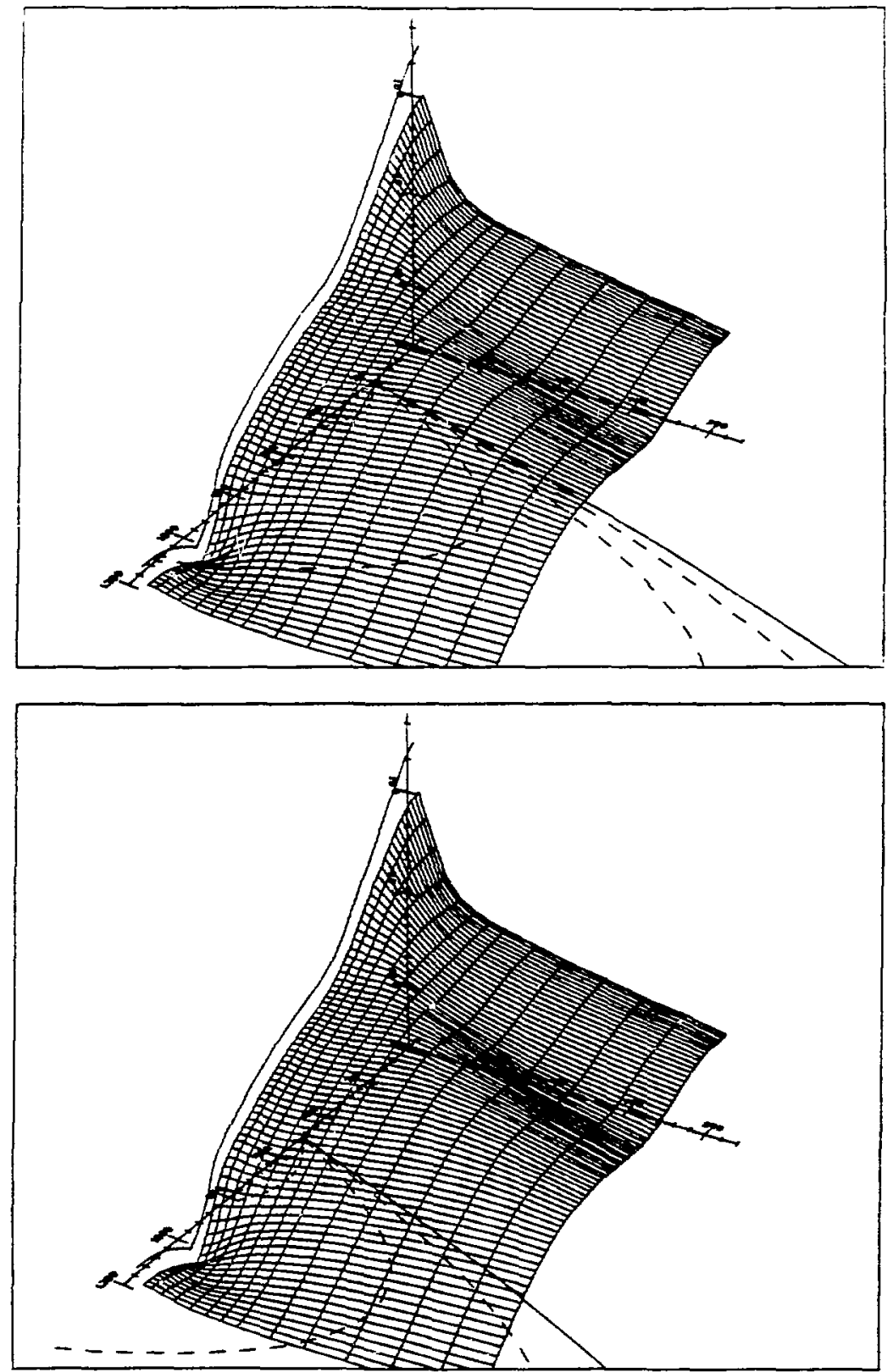

Figure 3: ${ }^{3} \mathrm{He}$ spectral function ( $T=0$ piece). The left aris is the momentum from 0 to $1200 \mathrm{MeV} / \mathrm{c}$ and the right axis is energy from 0 to $250 \mathrm{MeV}$. In the $(\mathrm{k}, \mathrm{E})$ plane are shown the integration region of Eq. 3 for $y=-.2 \mathrm{GeV} / \mathrm{c}$ (top) and $y=-0.5 \mathrm{GeV} / \mathrm{c}$ (bottom) for several momentum transfers. The solid line is $q=100 \mathrm{GeV} / \mathrm{c}$. Covering less of the $(\mathrm{k}, \mathrm{E})$ plane, in order, are the dashed lines indicating $q=2,1.5,1$, , and $0.5 \mathrm{GeV} / \mathrm{c}$ (for $y=-0.2 \mathrm{GeV} / \mathrm{c}$ only). 


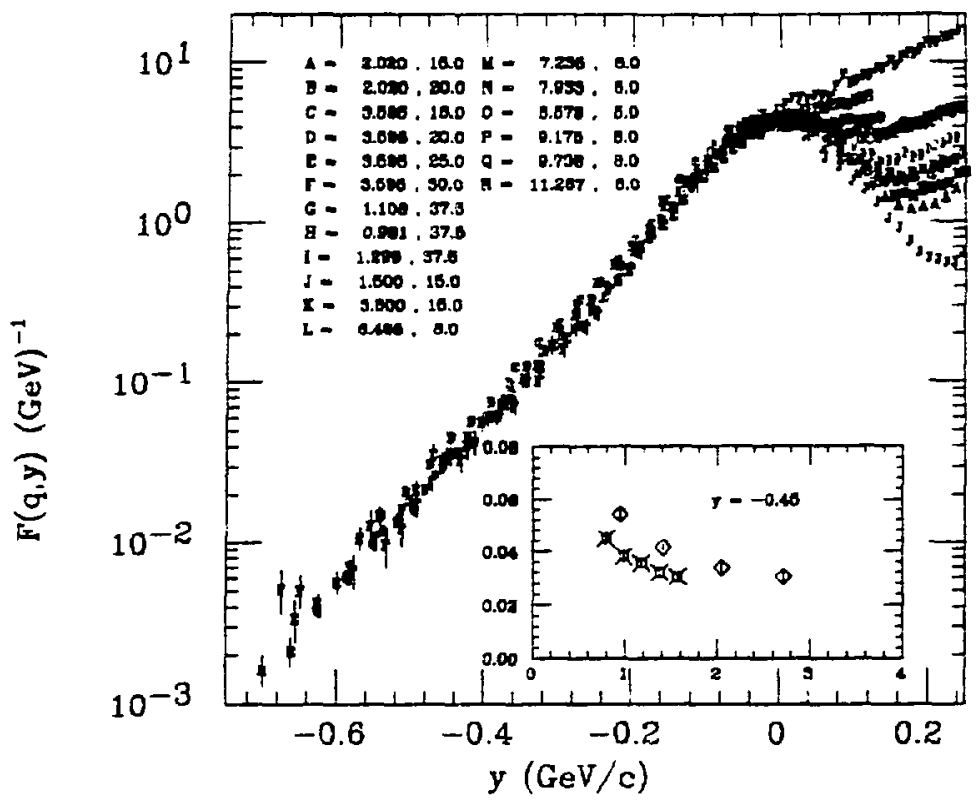

Figure 4: $F(q, y)$ for ${ }^{4} \mathrm{He}$. The insert shows the convergence of $F(q, y)$ with $Q^{2}$ for $y=$ $-0.45 \mathrm{GEV} / \mathrm{c}$. The diamonds represent data taken at $16^{\circ}, 20^{\circ}, 25^{\circ}$, and $30^{\circ}$, while the stars are for data at $8^{\circ}$. Data from Ref. $[10,11,12]$.

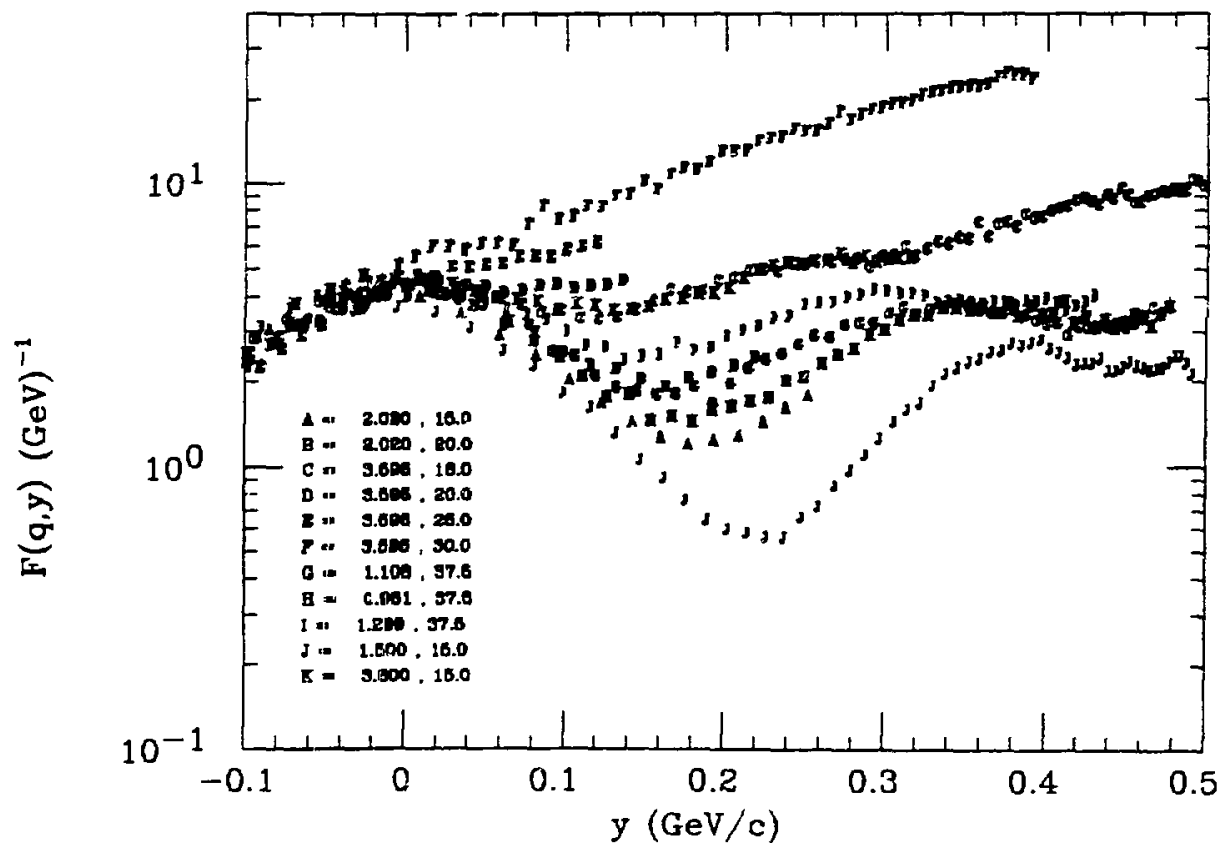

Figure 5: ${ }^{4} \mathrm{He}$ scaling function in the region of energy loss that includes the $\Delta$ peak. 


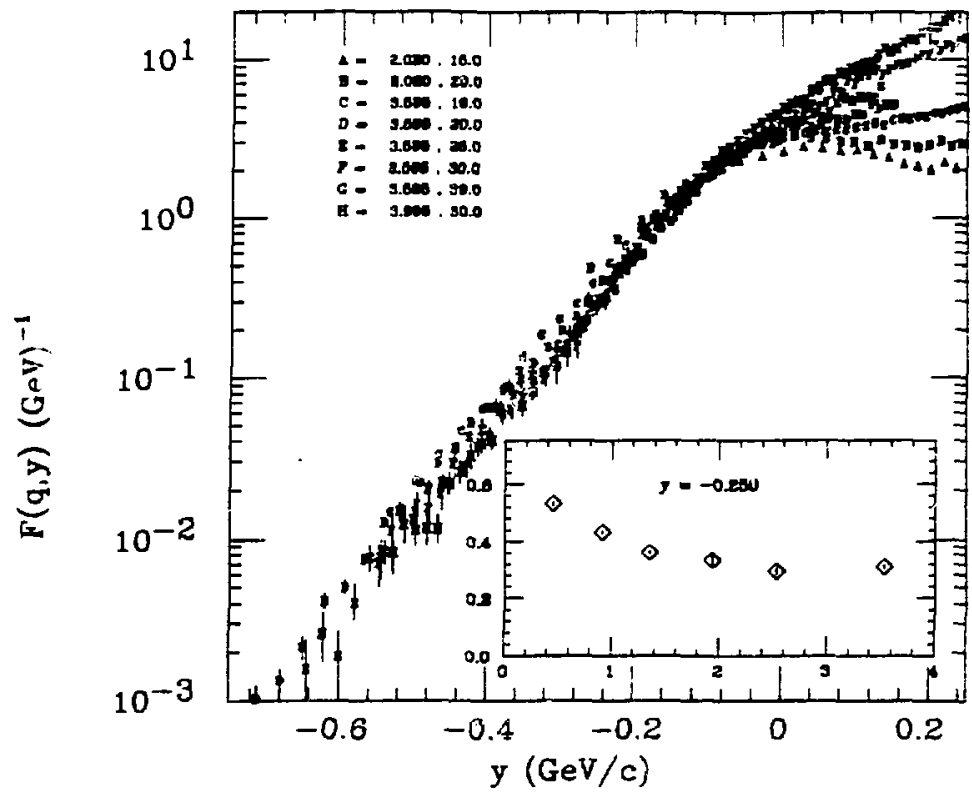

Figure 6: $\mathrm{F}(q, y)$ for $\mathrm{Fe}$. The insert shows the convergence of $\mathrm{F}(q, y)$ with $Q^{2}$ in $(\mathrm{GeV} / \mathrm{c})^{2}$ for $y=-0.25 \mathrm{GeV} / \mathrm{c}$. Data from Ref. [11].

also exhibit scaling but to a lesser degree. The role of FSI, which have been ignored up to this point, should be more important in heavy nuclei, and the increased fermi momentum has the effect of sweeping some of the inelastic contributions into the negative $y$ region. Iron is shown as an example in Figure 6.

The role of FSI between the knocked out nucleon and the $(A-1)$ residual system can be significant and may be the source of the longstanding disagreement between the ${ }^{3} \mathrm{He}$ PWIA results generated from Faddeev spectral functions and the data at high $q$ [13]. It is difficult to include the role of FSI in calculations and it would be surely preferable to avoid them. We show in Figure 7 for ${ }^{3} \mathrm{He}$ at $8^{\circ}[2]$ the relative energy of the knocked out nucleon and $(A-1)$ system in the center of mass as a function of momentum transfer assuming no internal excitation of the $(A-1)$ system. The solid lines indicate constant values of $y$ and the letters correspond to the existing ${ }^{3} \mathrm{He}$ data. If we assume that the FSI depend on the nucleon-nucleus total cross section which is a function of the relative $\mathrm{CM}$ energy, there is an advantage to go to large $q$. Note that none of the data from SLAC at large values of $|y| \geq 0.5 \mathrm{GeV} / \mathrm{c}$ yield large $\mathrm{CM}$ energies, indicating that they may be strongly influenced by FSI. In a recent publication Ciofi delgi Atti and coworkers [7] find that an expansion of $F(q, y)$ in inverse powers of $q$ allow an an asymptotic vaiue of $F(\infty, y)$ to extrapolated from finite $q$ data, avoiding FSI which they suggest should go as $\frac{1}{q}$. Consequently they would argue that it is important, for a scaling analysis, to have high quality data up to moderate values of $q$, say $3-4 \mathrm{GeV} / \mathrm{c}$, avoiding the experimental difficulties associated with asymptotic values of $q$.

We conclude that large initial nucleon momenta may probed through quasielastic scattering. A scaling analysis allows the extraction of the longitudinal momentum distribution 


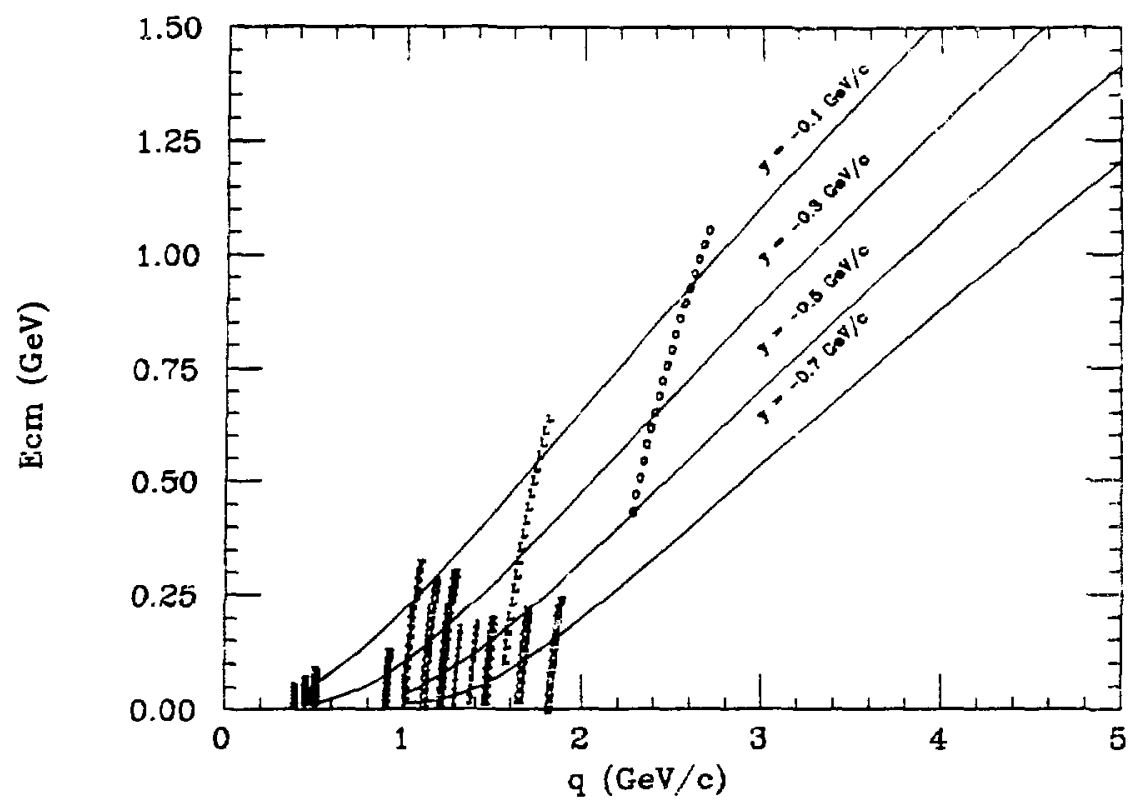

Figure 7: Relative energy of the knocked out nucleon and the (A-1) nucleus (assuming no internal excitation) as a function of momentum transfer. Solid line are for fixed values of $y$. Letters correspond to existing data on ${ }^{3} \mathrm{He}$ at $8^{\circ}$; ' $\mathrm{L}$ ' and ' $\mathrm{O}$ ' correspond to $10.95 \mathrm{GeV}$ and $14.7 \mathrm{GeV}$ respectively.

once the momentum transfer is large enough that the piece of the spectral function which is not accessible becomes negligible and the $\mathrm{CM}$ energies are large enough to minimize the effects of final state interactions.

\section{Deep Inelastic Scattering}

We now turn our attention to the piece of the inclusive spectrum that is due to process other than elastic scattering from a moving nucleon. We have shown, in Figure 2, the contribution of inelsistic nucleon processes to the spectrum of scattered electrons, and we can see in Figures 4, 5 and 6 that they fail to scale as the quasielastic piece. The reactions contributing to the inclusive spectrum are those seen in inelastic scattering from the free nucleon - real and virtual pion production, resonance production (including the $\Delta$ and the $\mathrm{N}^{*}$ ) and scattering from quarks inside the nucleon. Our goal in this section is simply to convince the reader that to a good approximation we can understand the DIS in terms of a conventional nuclear physics approach. Together with the $y$ scaling description of the previous section we can make some estimates for the relative contributions of quasielastic and deep inelastic scattering at high energies.

If we make the (probably correct) assumption that there are no exotic multiquarks states in nuclei we can calculate the nuclear deep inelastic scattering. There have been some recent reviews, Ref. [14] for example, and some new work [15] which provide all the necessary details which I omit here. 
The deep inelastic inclusive electron scattering cross section is written as

$$
\frac{d^{2} \sigma}{d E^{\prime}} \frac{d \Omega}{d \Omega}=\frac{4 \alpha^{2}}{q_{\mu}^{4}} E^{\prime 2}\left\{W_{2}^{(A)} \cos ^{2} \frac{\theta}{2}+2 W_{1}^{(A)} \sin ^{2} \frac{\theta}{2}\right\}
$$

We can write, following the work of Bodek and Ritchie $[16]$ and ignoring the ambiguities of the off-shell character of the struck nucleon,

$$
W_{1}^{A}=Z \int d^{3} \vec{P}|\phi(\vec{P})|^{2}\left(W_{1}^{p}+\frac{W_{2}^{p}}{2 M_{p}^{2}}\left(\vec{P}^{2}-P_{3}^{2}\right)\right)
$$

and

$$
W_{2}^{A}=Z \int|\phi(\vec{P})|^{2}\left[\left(1+\frac{P_{3} Q^{2}}{M_{p} \nu^{\prime} q_{3}}\right)^{2}\left(\frac{\nu^{\prime}}{\nu}\right)^{2}+\frac{\vec{P}^{2}-P_{3}^{2}}{2 M_{p}^{2}}\left(\frac{Q^{2}}{q_{3}^{2}}\right)\right] W_{2}^{p},
$$

where $|\phi(\vec{P})|^{2}$ is the nucleon momentum distribution, $P_{3}$ is the momentum component along $\vec{q}$ and $v^{\prime}=\frac{P_{i \cdot q}}{M_{n}}$. $W_{1,2}^{p}$ are the free proton inelastic structure functions. To $W_{1,2}^{A}$ in Eqs. 5 and 6 we must add similar terms for the neutron. With this formalization, it is possible, using a realistic nucleon momentum distribution to smear the nucleon inelastic structure functions so as to obtain the nuclear inelastic response. Despite its failure to properly estimate binding effects, the Bodek and Ritchie method remains, for our purposes, quite useful. It is possible to improve upon the Bodek and Ritchie approach by using a spectral function instead of a single particle momentum distribution. Other improvements have been considered [14, 15]. We give some results below for ${ }^{3} \mathrm{He}$ which were calculated using the spectral function of $[8]$.

In Figure 8, 9 and 10 we have reproduced the calculations of the full inclusive response using the spectral function of [8] along with the experimental data [2] at three different energies; $7.257 \mathrm{GeV}, 10.954 \mathrm{GeV}$, and $14.696 \mathrm{GeV}$, all at $8^{\circ}$. The dotted line is the quasielastic piece (Eq. 1), the dashed line is the inelastic nuclear response calculated using the parameterized inelastic nucleon responses found in [17]. The solid line is the sum of the two pieces. The agreement with the data is quite good, though not perfect. At low $\omega$ which is completely dominated by the quasielastic scattering, we see the longstanding discrepancy for ${ }^{3} \mathrm{He}$ which may be due to FSI. These calculations are useful in that they indicate that deep inelastic processes would heve to be many orders of magnitude greater before we could attribute any excess strength in the cross section at small $\omega$ to nucleon inelastic processes.

The region at $x>1$ is very sensitive to the nucleon momentum distribution since in this region the struck quark is carrying a momentum fraction greater than that available to the free nucleon. Any strength in the structure function at $x>1$ inust be due to the nucleon momentum in the nucleus. Several workers have begun to study this region and have found that the nuclear structure function at large values of $x$ is greatly enhanced due to short range correlations as compared to mean field momentum distribution, e.g. Fermi gas. As an example we show in Figure 11 the work of [18] who have calculated the deep inelastic structure function for ${ }^{56} \mathrm{Fe}$ and find inclusion of correlations have a significant effect. 


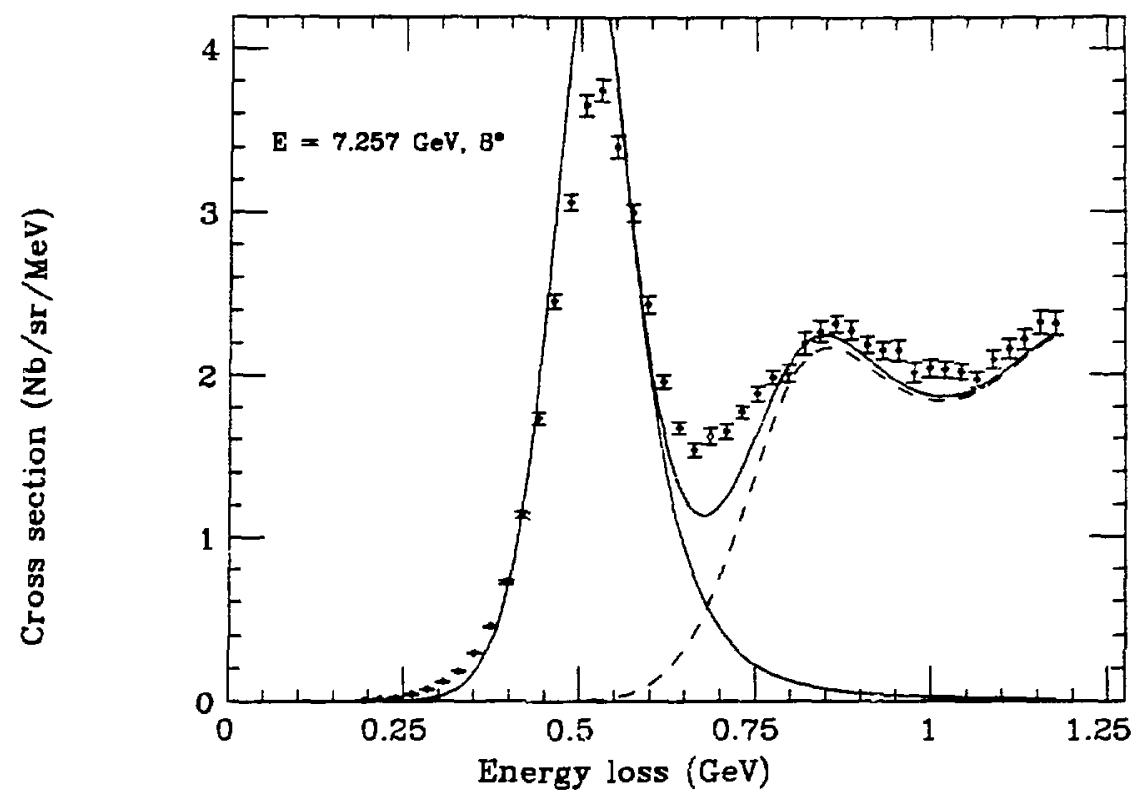

Figure 8: Experimental data on ${ }^{3} \mathrm{He}$ at $7.257 \mathrm{GeV}$ and $8^{n}$ along with model calculations of the quasielastic (dotted line), deep inelastic (dashed line) and the sum (solid line).

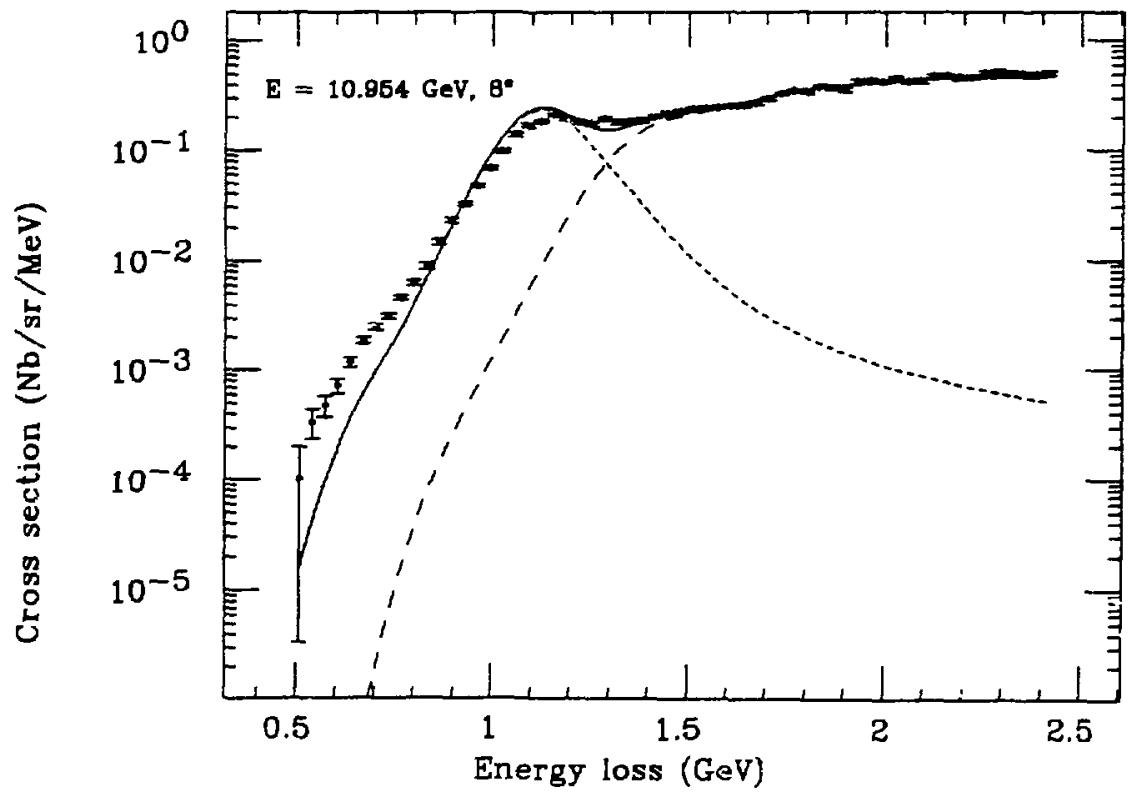

Figure 9: Experimental data on ${ }^{3} \mathrm{He}$ at $10.954 \mathrm{GeV}$ and $8^{\circ}$ along with model calculations of the quasielastic (dotted line), deep inelastic (dashed line) and the sum (solid line). 


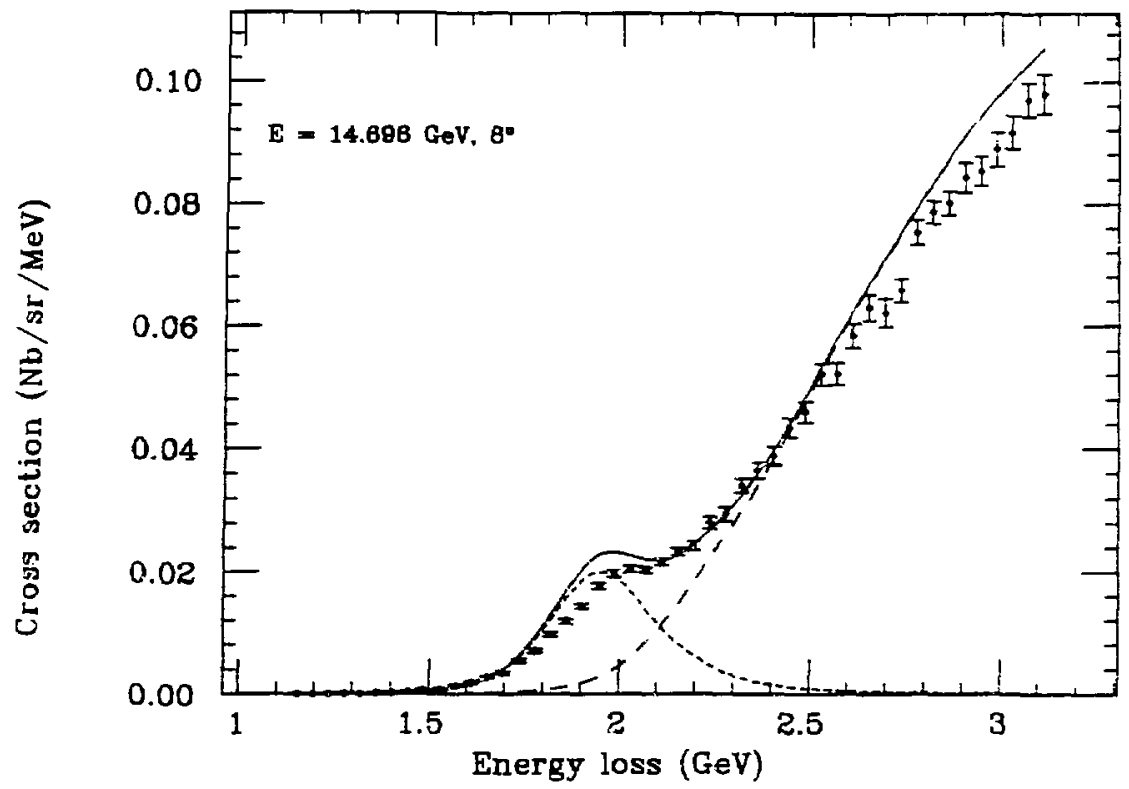

Figure 10: Experimental data on ${ }^{3} \mathrm{He}$ at $14.696 \mathrm{GeV}$ and $8^{\circ}$ along with model calculations of the quasielastic (dotted line), deep inelastic (dashed line) and the sum (solid line).

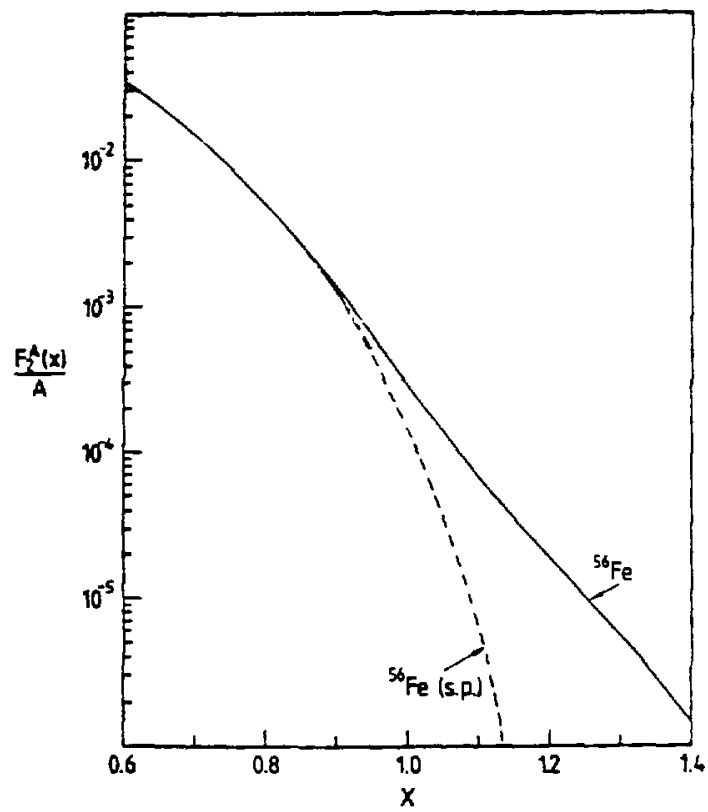

Figure 11: Deep inelastic structure functions for ${ }^{56} \mathrm{Fe}$ from Re; [18] The dashed line is a single particle model and the solid line results when correlations are included 


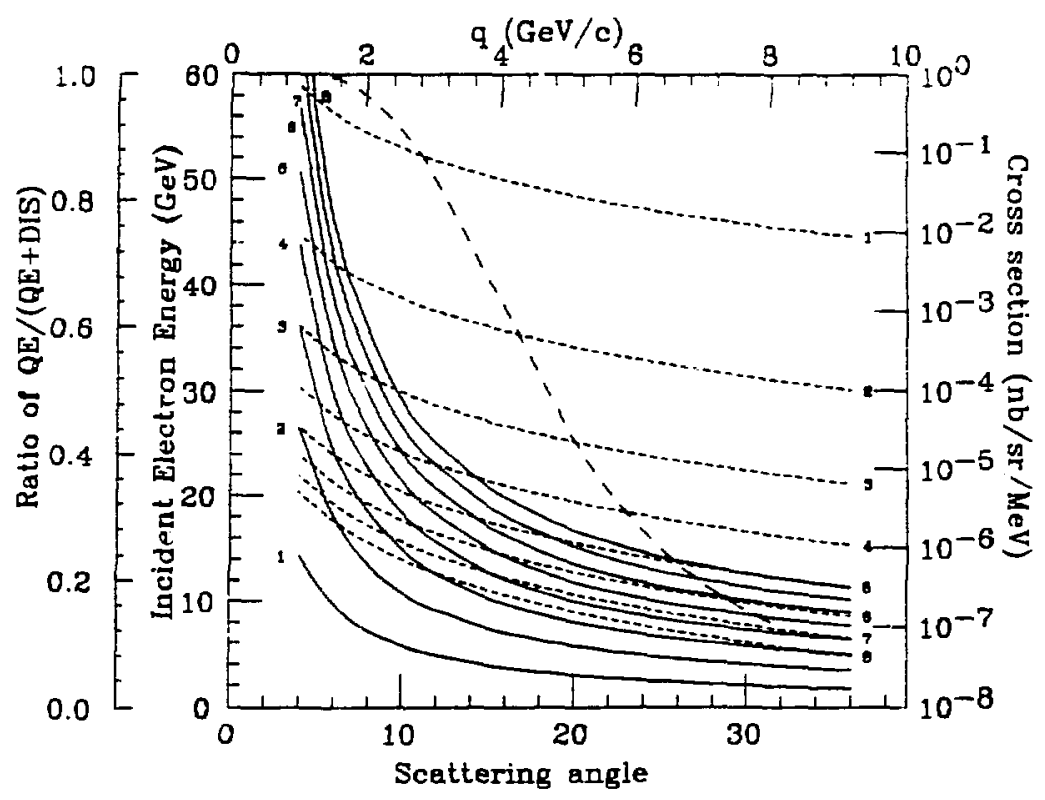

Figure 12: Kinematics, cross sections, and ratio of quasielastic scattering to total inclusive scattering for iron at $y=-0.5 \mathrm{GeV} / \mathrm{c}$. See text for explanation.

\section{Kinematics and Cross Sections}

With the basic formalism presented we can calculate cross sections for the inclusive scattering at high momentum transfer. We present the results of some calculations which can be used to determine at what momentum transfers it will be possible to measure the quasielastic and deep inelastic processes with a minimum of interference between the two. Recall that as the momentum transfer increases the quasielastic process dies out very quickly, at least as fast as the elastic form factor. At the same time the deep inelastic processes grow and at $x \cong 1$ they are dominate. Since the DIS cross section is a convolution of the inelastic structure functions which show little $q$ dependence, the limitation in going to larger values of $x$ is determined by the amount of high momentum components in the initial state.

The cross sections presented below have been calculated using, not a spectral function but a momentum distribution extracted from a scaling analysis of the quasielastic data at the highest momentum transfer. This was done for two reasons. The most obvious is that for heavy nuclei, spectral functions are not widely available and also our studies with ${ }^{3} \mathrm{He}$ indicate that all sensitivity to the energy integration discussed in Section 2 is removed by the necessarily large energy transfer in DIS. We make no attempt to treat the binding energy properly; nevertheless we believe these results are good to the $20 \%$ level. The same momentum distribution was used for the quasielastic and DIS parts of the response. We present results for iron although other nuclei could also have been chosen.

We have selected two different kinematics to emphasize the two different pieces of the response. The first is shown in Figure 12 for a fixed value of $y=-0.5 \mathrm{GeV} / \mathrm{c}$, where up to moderate $q$, quasielastic scattering dominates the response. Figure 13 is for a fixed 


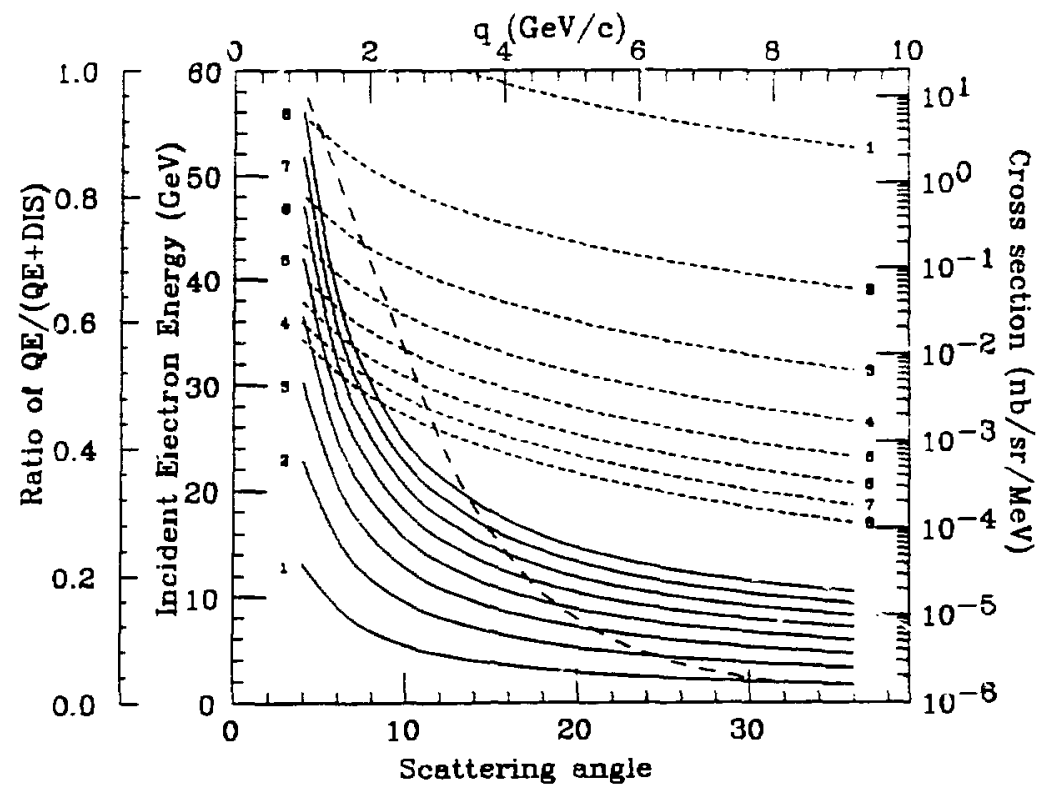

Figure 13: Kinematics, cross sections, and ratio of quasielastic scattering to total inclusive scattering for iron at $x=1$. See text for explanation.

value of $x=1$ where the spectrum very quickly becomes dominated by the DIS as $q$ increases. Some care must be made in explaining these plots as we have tried to put a lot of information in them. The solid lines which are labeled, from the bottom to the top, with the (three) momentum transfer ( 1 to 8 ), refer to the bottom axis (scattering angle) and the first left axis (the incident electron energy). From these solid lines one can simply read off the necessary electron energy to scatter at a fixed momentum transfer over the angular range indicated. (Keep in mind that for Figure 12, this is at a fixed value of $y=-0.5 \mathrm{GeV} / \mathrm{c}$.) The short dashed lines labeled, from top to bottom, with the (three) momentum transfer ( 1 to 8 ), refer to the scattering angle and the right axis - the total cross section in $\mathrm{nb} / \mathrm{sr} / \mathrm{MeV}$. These lines can be used to pick off the cross section at a fixed momentum transfer over the angular range of 4 to 36 degrees. The single long dashed line gives the ratio of the quasielastic cross section to the sum of the quasielastic and the DIS. This ratio is only a function of $q$ and should be read against the top axis and the far left axis.

These figures can be used to make count rate estimates and to set limits over the range of $q$ where it might be possible to study the two dominant pieces of the inclusive response. We can expect for a single arm experiment to handle a luminosity $\mathcal{L}=10^{37} \mathrm{~cm}^{-2} \mathrm{~s}^{-1}$. Together with a spectrometer of $\Delta \Omega=5(-3) \mathrm{msr}$ and a final energy bin of $50 \mathrm{MeV}$, these conditions would yield a count rate of $9(6)$ events per hour for a cross section of 1 $\mathrm{nb} / \mathrm{sr} / \mathrm{MeV}$. A reasonable lower limit for the count rate is $10 \mathrm{hr}^{-1}$ implies cross sections as low as $10^{-6} \frac{n b}{a r \mathrm{MeV}}$ could be measured. Obviously the higher the incident energy the further out in $q$ we can go.

To make an example, for $y=-0.5 \mathrm{GeV} / \mathrm{c}$ (from Figure 12 ), for $\sigma=10^{-6} \frac{n b}{\operatorname{srMeV}}$ we find that the $q=6 \mathrm{GeV} / \mathrm{c}$ short dashed line is intersected at about $\theta=16^{\circ}$. Looking at the 
solid line for $q=6 \mathrm{GeV} / \mathrm{c}$ at $16^{\circ}$ we see that it is necessary to have an incident energy of about $16 \mathrm{GeV}$. The scattered electron energy would be about $11.5 \mathrm{GeV}$. Despite the large $x$ value of this point, the quasielastic contribution to the total cross section would only be about $30 \%$. To make a cleaner measurem nt of the quasielastic piece would require going to lower $q$ and perhaps to lirger vaiues of $x$ (more negative $y$ ).

A measurement of the DIS contribution at very large $q($ at $x=1)$ would be much faster. From Figure 13 we see that even at $q=8 \mathrm{GeV}$ 'c the rates would be quite large (greater than $900 \mathrm{hr}^{-1}$ ), again requiring an incident electron energy of less than $20 \mathrm{GeV}$ and a scattered electron energy of about $10 \mathrm{GeV}$. This measurement would be virtually free of any quasielastic contribution: the elastic nucleon cross section has fallen by many orders of magnitude. We expect that it will possible to measure nuclear DIS at $x \geq 1$ out $q=10-12 \mathrm{GeV} / \mathrm{c}$.

Finally we should note the limitations of the SLAC and CEBAF to study this region. At SLAC sufficient beam energy is available but the luminosity is about two orders of magnitude less. At CEBAF the maximum beam energy is ": $\mathrm{GeV}$ and to reach the large $q$ we discuss here would require scattering at very backward angles where the cross sections are immeasurably small.

\section{Conclusion}

A new electron machine of high current and energies wiil significantly increase the range in momentum transfer that can be studied through inclusive electron scattering. In the region of $x \geq 1$ two dissimilar processes compete, quasielastic scattering and deep inelastic scattering. Despite having very different momentum dependences they both sample the same initial state nuclear structure. These experiments have the potential to reveal the short range behavior of the NN interaction by providing a measurement of the high $k$ components of the nuclear wave function. In addition they allow the study of the fundamental electron-nucleon interaction. Investigations of this type for a variety of nuclei will make an important contribution to the understanding the role of nuclear structure in quasielastic and DIS, thereby setting limits on the role of exotic states in the nucleus.

The main result of this paper is found in Section 4 where we present the results of calculations for the inclusive cross sections over a wide range of momentum transfer. Using $y$ scaling for quasielastic scattering and a convolution model for DIS we believe that these calculations are reliable and can be used to indicate the experimental program that could be studied with a machine capable of $20 \mathrm{GeV}$ and $200 \mu \mathrm{A}$. These predictions indicate that a measurement of the inclusive response can be made at very large momentum transfer in such a way as to isolate the DIS from the quasielastic. It is unlikely that these measurements will be possible without a new machine.

\section{References}

[1] T. de Forest Jr. Nucl. Phys., A132:305, 1969. 
2! D. Day, J.S. McCarthy. I. Sick. R.G. Arnoid. B.T. Chertok. S. Rock, Z.M. Szalata. F. Martin. B.A. Mecking, and G. Tamas. Phys. Rev. Lett.. 43:1143, 1979.

3 A. E. L. Dieperink, T. de Forest. I. Sick. and R. A. Brandenburg. Phys. Lett., B63:261. 1976 .

4] T. de Forest Jr. Vucl. Phys., A392:232. 1983.

'5: G. B. West. Phys. Rep., 18:263, 1975.

6! D. Day, J.S. McCarthy. T.W. Donnelly, and I. Sick. Ann. Rev. of Vucl. Part. Sci.. $40: 35$ T. 1990.

iT) C. Ciofi degli Atti, E. Pace, and G. Salme. Phys. Rev., C, 1991.

8] H. Meier-Hadjuk, Ch. Hajduk, P. U. Sauer, and W. Theis. Nucl. Phys.. A395:332, 1983.

9! C. Ciofi degli Atti. E. Pace, and G. Salme. Phys. Rev., C39:259, 1989.

\{10! S. Rock. R. G. Arnold, B. T. Chertok. Z. M. Szalata, D. Day, J. S. McCarthy. F. Martin. B. A. Mecking, I. Sick, and G. Tamas. Phys. Rev., C26:1592, 1982.

(11) D. Day, J.S. McCarthy, Z.E. Meziani, R. Minehart, R. Sealock, S.T. Thornton, J. Jourdan, I. Sick, B.W. Filippone, R.D. McKeown, R.G. Milner, D.H. Potterveld, and Z. Szalata. Phys. Rev. Lett., 59:427, 1987.

[12] R. M. Sealock, K. L. Giovanetti, S. T. Thornton, Z. E. Meziani, O.A. RondonAramayo, S. Auffret, J.-P. Chen, D. G. Christian, D.B. Day, J.S. McCarthy, R. C. Minehart, L. C. Dennis. K. W. Kemper. B. A. Mecking, and J. Morgenstern. Phys. Rev. Lett., 62:1350, 1989.

[13] H. Meier-Hadjuk, U. Oelfke, and P. U. Sauer. Nucl. Phys., A499:637, 1989.

[14] R.P. Bickerstaff and A.W. Thomas. J. Phys G: Nucl. Part. Phys., 15:1523, 1989.

15] C. Ciofi degli Atti and S. Liuti. Phys. Rev., C41:1100, 1990.

[16] A. Bodek and J. L. Ritchie. Phys. Rev., D23:1070, 1981.

(17] A. Bodek, M. Breidenbach, D.L.Dubin, J.E. Elias, J.I. Friedman, H. W. Kendall, J.S.Poucher, E.M. Riordan, M.R. Sogard, D.H. Coward, and D.J. Sherden. Phys. Rev., D20:1471, 1979.

[18] J. Rozynek and M.C. Birse. Phys. Rev., C38:2201, 1988. 